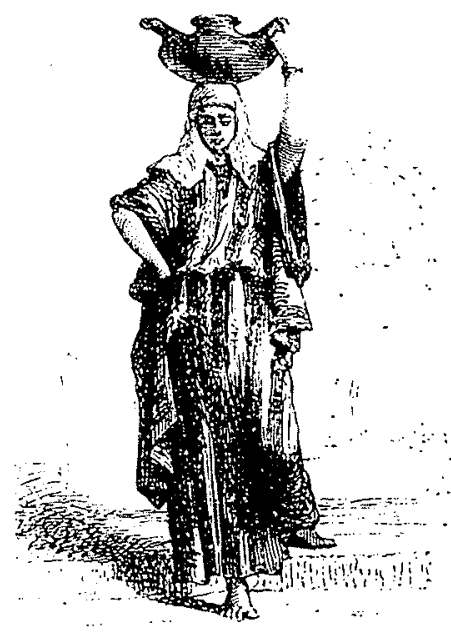

\title{
LE BILAN DE MASSE DE L'INDLANDSIS DU GROENLAND N'EST PAS POSITIF
}

\section{PAR A. BAUER *}

Le bilan de masse de l'ensemble de l'Indlandsis du Groenland demande la connaissance des surfaces d'accumulation et d'ablation, de la valeur moyenne de l'accumulation et de l'ablation, et de la perte de masse sous forme d'icebergs par vêlage des glaciers effluents de l'Indlandsis.

Dans notre première tentative pour chiffrer le bilan de masse (Bauer, 1954), nous avions donné le détail des hypothèses sur l'altitude de la ligne d'équilibre pour les différentes zones de l'Indlandsis correspondant aux quinze feuilles au $1 / 1000000^{\circ}$ de la World Aeronautical Chart, et cela après avoir précisé le contour de l'Indlandsis. Ces hypothèses se basaient sur l'ensemble de la littérature existante.

Nous en déduisons les valeurs suivantes:

Surface de la zone d'accumula- $1439800 \mathrm{~km}$ z $\quad 83,5 \%$

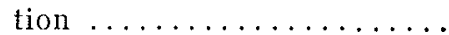

Surface de la zone d'ablation $286600 \mathrm{~km}^{2} \quad 16,5 \%$ Surface totale de l'Indlandsis $\overline{1726400 \mathrm{~km}^{2}} \overline{100,0 \%}$

Comme valeur de l'accumulation moyenne pour l'ensemble de l'Indlandsis, nous adoptions la valeur de F. Loewe, soit $0,31 \mathrm{~m}$ valeur en eau, la meilleure estimation alors disponible. Pour l'ablation moyenne, nous prenions celle du profil étudié de la zone d'ablation à l'est de la Baie de Disko, soit $1,1 \mathrm{~m}$ valeur en eau, et cela pour l'ensemble de la zone d'ablation de l'Indlandsis du Groenland.

A ces valeurs correspondent :

- pour l'accumulation ... $446 \mathrm{~km}^{3} /$ an valeur en eau - pour l'ablation ....... $315 \mathrm{~km}^{3} / \mathrm{an}$ valeur en eau

Pour la perte de masse sous forme d'icebergs, nous avions donné le détail du vêlage des glaciers des différentes parties des côtes du Groenland. A partir de la largeur du front des glaciers, de leur

* Centre d'Etudes Glaciologiques des Régions Arctiques et Antarctiques. vitesse moyenne connue ou estimée et de leur épaisseur moyenne connue ou estimée, nous obtenions un débit total de $240 \mathrm{~km}^{3}$ de glace par an, soit $215 \mathrm{~km}^{3} /$ an valeur en eau.

Le bilan de masse de l'Indlandsis était done: $446+(-315-215)=-84 \mathrm{~km}^{3} /$ an valeur en eau, soit un bilan négatif d'environ $100 \mathrm{~km}^{3}$ de glace par an. Vu l'incertitude avec laquelle étaient connues les données utilisées, le résultat ne donnait qu'un ordre de grandeur. Dans son ensemble, l'Indlandsis du Groenland apparaissait en faible décrue, comparativement à son volume total de 2,6 millions de $\mathrm{km}^{3}$ de glace ou 2,35 millions de $\mathrm{km}^{3}$ valeur en eau.

\section{Précision \\ sur les données du nord et du centre de IIndlandsis du Groènland}

Les travaux parus depuis cette première tentative (Benson, 1960) fournissent des données plus précises sur les parties nord et centrale de l'Indlandsis du Groenland, en particulier en ce qui concerne l'altitude de la ligne d'équilibre dans le nord, donc sur la surface respective d'accumulation et d'ablation. La valeur moyenne pour l'ensemble de l'Indlandsis s'établit à $0,34 \mathrm{~m}$ valeur en ear pour l'accumulation.

Les valeurs correspondantes sont les suivantes: Surface d'accumulation ..... $1471800 \mathrm{~km}^{2} \quad 85 \%$ Surface d'ablation .......... $\quad 254600 \mathrm{~km}^{2} \quad 15 \%$ Surface totale (inchangée) .. $1726400 \mathrm{~km}^{2} \quad 100 \%$

\section{Calcul du bilan de masse de H. Bader}

Dans le bilan de masse de l'Indlandsis du Groenland publié par C.R.R.E.L. (Bader, 1961), l'auteur utilise les données de A. Bamer et de C. Benson. Il faut cependant faire les remarques suivantes.

En partant de la carte du Groenland comportant 
les courbes d'égale précipitation, H. Bader obtient une précipitation totale de $636 \mathrm{~km}^{3} /$ an valeur en eau pour tout l'Indlandsis. De plus, il établit le total des précipitations pour la zone d'ablation en dessous de la ligne d'équilibre, soit $122 \mathrm{~km}^{3} /$ an valeur en eau. Or, dans le calcul du bilan, il prend comme accumulation la valeur des précipitations totales, soit $636 \mathrm{~km}^{3} / \mathrm{an}$, ce qui revient à admettre une accumulation moyenne, pour la zone d'accumulation, de $0,43 \mathrm{~m} / \mathrm{m}^{2} /$ an. C'est une erreur, car il faut soustraire des précipitations totales celles de la zone d'ablation. En effet, celles-ci fondent en été et n'entrent pas dans le calcul du bilan qui est en en réalité de $636-122=514 \mathrm{~km}^{3} /$ an valeur en eau, précipitations résiduelles appelées accumulation.

En ce qui concerne l'ablation, H. Bader cite les estimations de A. Bauer et de C. Benson, soit respectivement 315 et $306 \mathrm{~km}^{3} / \mathrm{an}$ valeur en eau. Mais il estime lui-même l'ablation comprise entre 120 et $270 \mathrm{~km}^{3} /$ an sous prétexte que, dans le nord, l'Indlandsis est en crue. Cela est hypothétique car, dans cette région, il n'a jamais été fait de mesures de l'ablation et encore moins de la décharge des glaciers sous forme d'icebergs. Or, d'une part, les observations récentes montrent que l'ablation est plus forte qu'on ne le pensait (Davies et Krinsley, 1962): le glacier Moltke a reculé en effet de $5 \mathrm{~km}$ en 1932 et 1959; d'autre part, pour ces mêmes raisons, on ne peut affirmer que l'Indlandsis de la région de Thule soit en augmentation. Même si c'était le cas, il faudrait encore prouver la dépendance entre le lobe de Thule et l'ensemble de l'Indlandsis. Dans tous les cas, il est difficile d'étendre les résultats concernant cette région limitée à tout le nord de l'Indlandsis du Groenland.

Pour la perte de masse sous forme d'icebergs, H. Bader utilise dans le calcul du bilan la valeur de $240 \mathrm{~km}^{3} /$ an valeur en eau, alors qu'il s'agit là de notre propre estimation d'un volume de glace auquel correspond $215 \mathrm{~km}^{3} /$ an valeur en eau.

Il s'en suit que le bilan établi doit être rétabli avec des valeurs justifiées.

\section{Nouveau calcul du bilan de masse de l'Indlandsis du Groènland}

Nous conservons la valeur moyenne de l'accumulation de $C$. Benson $\left(0,34 \mathrm{~m} / \mathrm{m}^{2} /\right.$ an valeur en eau) confirmée par les recherches dans la partie centrale de l'Indlandsis par l'Expédition Glaciologique Internationale au Groenland en 1959. Nous conservons aussi les surfaces d'accumulation et d'ablation de l'Indlandsis du Groenland citées.

Pour la valeur de l'ablation moyenne, nous adoptons encore celle mesurée avec précision sur un profil à l'est de la Baie de Disko. Ce profil, de latitude et d'altitude moyennes, est pris comme profil type du Groenland. La valeur moyenne, obtenue d'une façon précise au cours de l'E.G.I.G. (Bauer, 1960 ), est de $1,30 \mathrm{~m} / \mathrm{m}^{2}$ /an valeur en eau (Ambach, 1963). Il faut ajouter que l'ablation a toujours été sous-estimée. Il a été démontré, en particulier, que l'ablation ne diminuait pas linéairement avec l'altitude, mais présentait un maximum relatif vers $1000 \mathrm{~m}$ d'altitude par suite de conditions de défla- tion générale dans cette zone. Ces conditions doivent être générales pour l'ensemble de la zone d'ablation de l'Indlandsis au vu de la surface des lacs de fonte qui passe par un maximum relatif à cette altitude.

En ce qui concerne la perte de masse sous forme d'icebergs par vêlage des glaciers émissaires de l'Indlandsis, le débit total est à reconsidérer.

Dans notre estimation antérieure, nous chiffrions le débit total à $240 \mathrm{~km}^{3}$ de glace par an et à $90 \mathrm{~km}^{3}$ de glace par an le débit solide des glaciers de la côte ouest au sud de la Baie de Melville.

Les mesures effectuées en 1957 et en 1958 au cours de l'E.G.I.G. (Bauer, 1966, a), renforcées par celles de 1964 (Bauer, 1966, b), montrent que les estimations anciennes sont trop faibles. Déjà seuls les vingt glaciers qui vêlent dans les Baies de Disko et de Umanak ont un débit solide total de $105 \mathrm{~km}^{3}$ de glace contre $80 \mathrm{~km}^{3}$ de glace d'après notre estimation antérieure. Si l'on extrapole cette augmentation du débit solide à l'ensemble des glaciers émissaires de l'Indlandsis, on obtient un débit solide total de $310 \mathrm{~km}^{3}$ de glace par an, soit $280 \mathrm{~km}^{3} /$ an valeur en eau.

Le nouveau calcul du bilan de masse s'établit en conséquence comme suit (valeur en eau) :

\begin{tabular}{|c|c|c|c|}
\hline & $\mathrm{km}^{2}$ & $\mathrm{~m} / \mathrm{m}^{2} / \mathrm{an}$ & $\mathrm{km} / \mathrm{an}$ \\
\hline Accumulation & 1471800 & $+0,34$ & +500 \\
\hline $\begin{array}{l}\text { Ablation } \ldots \ldots \ldots \ldots \ldots \\
\text { Débit des glaciers }\end{array}$ & 254600 & $-1,30$ & -330 \\
\hline Bilan de masse ... & & & -110 \\
\hline
\end{tabular}

Ce bilan de $-110 \mathrm{~km}^{3} /$ an valeur en eau représente une tranche d'eau de $+0,3 \mathrm{~mm} /$ an répartie uniformément sur l'ensemble des mers du globe.

Sans oublier l'incertitude des valeurs utilisées liée aux hypothèses de travail, aux extrapolations faites des valeurs moyennes à l'ensemble de l'Indlandsis du Groenland, il n'en reste pas moins, avec les réserves exprimées, que l'Indlandsis est finalement stationnaire ou en décrue. Dans tous les cas, le bilan de masse de l'Indlandsis du Groenland n'est pas positif.

\section{Nouveaux indices de décrue de l'Indlandsis du Groènland}

Il existe de nouvelles données de la décrue générale de l'Indlandsis du Groenland.

L'ensemble des glaciers de la côte ouest est en recul constant (Weidick, 1959).

Le glacier de l'Eque (Bauer, 1955), dont le front a été couvert de photographies aériennes en 1957 et en 1964, montre une tendance de décrue, depuis l'avance limitée de 1920 à laquelle ont aussi participé le glacier de Jakobshavn et le glacier de Moltke.

L'ensemble des glaciers du nord du Groenland est en décrue (Davies and Krinsley, 1962). Cette décrue s'accélère depuis 1946 (Mock, 1966). Il en est de même des glaciers du nord-est du Groenland (Wyllie, 1956).

Le glacier de Jakobshavn, qui draine une grande partie des glaces de l'Indlandsis (Bauer, 1954), a vu son front reculer d'une vingtaine de kilomètres depuis environ cent ans. L'épaisseur du front actuel a diminué d'environ $300 \mathrm{~m}$ et cette diminution a 
entrainé une diminution progressive de son bassin de drainage. On peut estimer à environ $500 \mathrm{~km}^{3}$ en cent ans, soit environ $5 \mathrm{~km}^{3}$ de glace par an, la perte supplémentaire de l'Indlandsis à l'est de la baie de Disko due à ce seul glacier (débit solide actuel du glacier de Jakobshavn : $36 \mathrm{~km}^{3}$ de glace par an). De même, le glacier d'Upernavik, pour la même période, a subi un recul analogue (Weidick, 1958).

La mesure répétée de figures de déformation peuvent fournir la variation actuelle d'un indlandsis d'après les travaux de $P$. Shumsky (Bauer, 1965). L'utilisation des résultats de telles mesures effectuées par E.G.I.G. en 1959 et en 1960 a donné pour l'ensemble de l'Indlandsis du Groenland une diminution de $50 \mathrm{~g} / \mathrm{cm}^{2} / \mathrm{an}$, soit au total une diminution d'environ $900 \mathrm{~km}^{3} /$ an valeur en eau (Shumsky, 1965).

Les observations de M. Koglbauer au Nunatak Scheideck $\left(71^{\circ} 11^{\prime} \mathrm{N}, 51^{\circ} 07^{\prime} \mathrm{W}, 950 \mathrm{~m}\right)$ ont montré que le bord de l'Indlandsis a diminué de $6 \mathrm{~m}$ de 1931 à 1961 (Loewe, 1964). Cette diminution de $0,20 \mathrm{~m} / \mathrm{an}$ de glace, compte tenu de la latitude et de l'altitude, est du même ordre de grandeur que celle que nous avons mesurée au bord ouest de l'Indlandsis par $69^{\circ} 40^{\prime} \mathrm{N}$ (Bauer, $1966, c$ ).

La répétition par E.G.I.G. en 1959 de la triangulation et du nivellement géodésique de 1948 du profil à la latitude moyenne de $69^{\circ} 40^{\circ} \mathrm{N}$ sur le versant ouest de l'Indlandsis, a mis en évidence pour l'ensemble de ce profil un abaissement moyen de $0,30 \mathrm{~m} /$ an valeur en eau. Comme pour l'ablation, si nous extrapolons cette valeur à l'ensemble de la zone d'ablation de l'Indlandsis, nous obtenons une diminution de masse de $76 \mathrm{~km}^{3} /$ an valeur en eau, du même ordre de grandeur que le bilan de masse négatif de l'Indlandsis obtenu par d'autres méthodes.

\section{Conclusion}

Il n'existe que des indications témoignant de la décrue générale de l'Indlandsis du Groenland. Il est possible que l'Indlandsis soit en crue, alor's que toutes les zones marginales sont en décrue : encore faut-il le prouver. Il est difficile d'affirmer que, dans le nord du Groenland en particulier, le bilan est positif alors que jamais des mesures d'ablation et de débit de glaciers ont été faites.

Il n'en reste pas moins que les données dont nous disposons actuellement, avec les hypothèses raisonnables formulées, nous conduisent à affirmer que le bilan de masse de l'Indlandsis du Groenland n'est pas positif. Il est négatif, avec des valeurs extrêmes comprises entre -100 et $-900 \mathrm{~km}^{3}$ de glace par an, soit une lame d'eau comprise entre $+0,3$ et $+2,5 \mathrm{~mm} /$ an répartie uniformément sur l'ensemble des mers du globe.

Des mesures futures préciseront ces données. En 1967 et en 1968, l'E.G.I.G. va procéder à la répétition des mesures effectuées en 1959 le long d'un profil traversant l'Indlandsis du Groenland dans sa partie la plus active. La variation de la position des sommets de la chaîne géodésique et la variation d'altitude des repères déterminée par nivellement géométrique, ainsi que la mesure répétée des figures de déformation, donneront une réponse à notre problème.

\section{Bibliographie}

Ambacr (W.), 1963. - Untelsuchungen zum Energieumsatz in der Ablationszone des Grönländischen Inlandeises (Camp IV-EGIG, 69 $40^{\circ} 05^{\prime \prime} \mathrm{N}, 40^{\circ} 37^{\prime} 58^{\prime \prime} \mathrm{W}$ ). Meddelelser om Gronland, Bd 174, Nr. 4, pp. 1-312.

Bader (H.), 1961. - The Greenland Ice Sheet. U.S. Army Cold Regions Research and Engineering Laboratory, I-B 2 , pp. 1-18.

Baver (A.), 1954. - Contribution à la connaissance de l'Indlandsis du Groenland: Synthèse glaciologique. Assemblée générale de Rome, A.I.H.S., no 39, pp. 270-296.

Baver (A.), 1955. - Le Glacier de l'Eqe. Actualités Scientifiques et Industrielles, $\mathrm{n}^{\circ} 1225$, pp. 1-120.

Bauer (A.), 1960. - Précision des mesures d'ablation. Assemblée Générale d'Helsinki, A.I.H.S., n ${ }^{\circ}$ 54, pp. 136143.

Bauen (A.), 1961. - Photogrammétrie et glaciologie E.G.I.G. Bulletin de la Société Française de Photogrammétrie, $\mathrm{n}^{\circ} 3$, pp. 3-24.

Bauen (A.), 1965. - Travaux du Groupe de Glaciologie de la IX ${ }^{\mathrm{e}}$ Expédition Antarctique Soviétique (été austral 19631964). Mémoires et Travaux de la Société Hydrotechnique de France, $\mathrm{n}^{\circ}$, pp. 115-124.

Bauer (A.), 1966, a. - Missions aériennes de reconnaissance au Groenland 1957-1958. Observations aériemnes et terrestres. - Exploitation des photographies aériennes. Détermination des vitesses des glaciers vêlant dans les Baies de Disko et de Umanak, E.G.I.G., vol. 2, no 1 (à paraître dans Meddelelser om Gronland).

BAUER (A.), 1966, b. - Exploitation des couvertures photographiques aériennes répétées du front des glaciers vêlant dans les Baies de Disko et de Umanak. - Institut de Géodésie de Danemark 1964. I : Nouvelles mesures photogrammétriques de la vitesse superficielle des glaciers du Groenland (Institut Géographique National, Paris). II : Accélération de l'écoulement des glaciers groenlandais vers leur front et determination de leur débit solide (A. Baner). E.G.I.G., vol. $2, n^{\circ} 3$ (à paraître dans Meddelelser om Gronland).

Bauer (A.), 1966, c. - Mouvement et variation d'altitude de la zone d'ablation ouest (latitude moyenne $69^{\circ} 40^{\prime} \mathrm{N}$ ) de I'Indlandsis du Groenland entre 1948 et 1959 . E.G.I.G. vol. 4, $\mathrm{n}^{\circ} 1$ (à paraître dans Meddelelser om, Gronland).

Brison (C. S.), 1960. - Stratigraphic studies of the snow and firm of the Greenland ice sheet. PhD Thesis, California Institute of Technology.

Davies (W. N.) and Kninsley (D.B.), 1962. - The recent regimen of the Ice Cap margin in North Greenland. Colloque d'Obergurgl, Variations du régime des glaciers existants, A.I.H.S., no 58, pp. 119-130.

LOEwe (F.), 1964. - Das Grönländische Inlandeis nach neuen Feststellungen. Erkunde, Bd XVIII, Lfg 3, pp. 189202.

Mock (S. J.), 1966. - Fluctuations of the terminus of the Molthe Glacier, Greenland. Journal of Glaciology (in print).

Shumskx (P.A.), 1965. - Ob ismenenii masei lednikovogo pokrova y zentre Grenlandii. Dokladi Aliademii Nauk SSSR, t. $162, \mathrm{n}^{\circ} 2$, pp. $320-322$.

WeIdick (A.), 1958. - Frontal variations at Upernivils Isstrom in the last 100 years. Meddelelser fra Dans. Geologik Forening, Bd 14, H. 1, pp. 52-60.

Werdrck (A.), 1959. - Glacial variations in West Greenland in historical time. Meddelelser om Gronland, Bd 158, Nr. 4 , pp. 1-196.

Wyllie (P. J.), 1956. - Ice recession in Dronning Louise Land, North-East Greenland, Journal of Glaciology, vol. 2 , pp. $704-708$. 